\title{
Alfabetización climática: actitudes y conocimientos del alumnado y profesorado de ciencias sociales
}

\author{
Gonzalo Pardo-Beneyto \\ Departamento de Derecho Constitucional, Ciencia Política y de la Administración. \\ Universitat de València. Valencia, España.gonzalo.pardo@uv.es \\ ORCID: https://orcid.org/0000-0001-7142-1740 \\ María Angeles Abellán-López \\ Departamento de Sociología y Antropología Social/Instituto Universitario de \\ Creatividad e Innovaciones Educativas. Universitat de València. Valencia, España. \\ maria.a.abellan@uv.es \\ ORCID: https://orcid.org/0000-0002-6553-0227
}

[Recibido: 12 junio 2021. Revisado: 15 diciembre 2021. Aceptado: 21 diciembre 2021]

Resumen: El cambio climático constituye una amenaza para la salud de la sociedad. Cada vez con mayor frecuencia las diversas organizaciones elaboran informes y programas sobre alfabetización climática. De hecho, las instituciones educativas están asumiendo esta responsabilidad incluyendo en las guías docentes la sostenibilidad curricular. Para evaluar el nivel conocimientos y percepciones del alumnado y profesorado de ciencias sociales se diseñó una encuesta exploratoria para determinados grupos en la Universidad de Valencia y en la Universidad de Alicante. El cuestionario contenía preguntas sobre cultura climática, actitudes y hábitos e incluía, además, un bloque de ítems sobre la relación entre cambio climático y la crisis sanitaria generada por la Covid-19.

Esta investigación generó tres hipótesis de trabajo y los hallazgos demostraron, con una tasa de respuesta del 59,2\%, que las actuaciones climáticas tienen más éxito cuando se abordan desde el comportamiento individual. Un porcentaje mayoritario de las personas encuestadas posee cierto nivel de cultura climática, está comprometido con hábitos sostenibles y adopta comportamientos responsables en la gestión de residuos. Sin embargo, no participa en las acciones reivindicativas para la lucha contra el cambio climático. Respecto a la relación entre cambio climático y Covid 19, una mayoría de las personas encuestadas señaló la existencia de una estrecha relación entre ambos fenómenos, si bien las respuestas ubicadas en las posiciones intermedias de la escala aumentaron significativamente.

Palabras clave: Cambio climático, cultura climática, alfabetización, gases efecto invernadero, conciencia ambiental.

\section{Climate literacy: attitudes and knowledge of students and teachers of social science}

Abstract: Climate change constitutes a threat to the health of society. More and more different organizations are developing reports and programs on climate literacy and culture. Likewise, educational institutions are assuming this responsibility in their teaching guides, including curricular sustainability. To assess the level of knowledge and perceptions of students and teachers of social sciences, an exploratory survey was designed for certain groups at the University of Valencia and the University of Alicante (Spain). The questionnaire contained questions on climate culture, attitudes and habits and a block of questions on the relationship between climate change and the Covid 19 health crisis. In addition, four working hypotheses were generated.

The main findings are, with a response rate of $59,2 \%$, that climate actions are more successful when approached from individual behavior to arrive at a collective vision as a common good. So, a majority percentage of the people surveyed possess a certain level of climate culture. Also, they are committed to healthy and sustainable habits, they adopt responsible behaviors of consumption and waste management. But they do not participate in the protest actions for the fight against climate change. Regarding the relationship between climate change and Covid 19, a majority of those surveyed 
indicate the existence of a close relationship between both phenomena. However, the responses that are located in the intermediate positions of the scale increased significantly.

Keywords: Climate Change, Climate Culture, Literacy, Greenhouse Gases, Environmental Awareness.

Para citar este artículo: Pardo Beneyto, G. \& Abellán López, M.A. (2021) Alfabetización climática: actitudes y conocimientos del alumnado y profesorado de ciencias sociales. Revista de Educación Ambiental y Sostenibilidad 3(2), 2603. doi: 10.25267/Rev_educ_ambient_sostenibilidad.2021.v3.i2.2603

\section{Introducción}

Según el Panel Internacional de Naciones Unidas sobre Cambio Climático (UNIPCC), uno de los grandes desafíos de la humanidad es el cambio climático porque tiene efectos generalizados sobre la vida humana y los ecosistemas. Por cambio climático se entiende un cambio en el estado del clima a lo largo del tiempo, ya sea debido a la variabilidad natural o como resultado de la actividad humana Así, la preocupación por el calentamiento climático antropogénico se ha instalado en la agenda global y sugiere un interés por identificar nuevas acciones sostenibles para afrontar el futuro. La magnitud y frecuencia del cambio climático impactan a través del aumento de olas de calor, inundaciones, aumento del nivel del mar, pérdida de biodiversidad, sequías o inundaciones y provocan pérdidas de vidas humanas, económicas y ambientales irreparables. Sus efectos han sido sentidos por numerosas comunidades que han quedado expuestas a diversas vulnerabilidades con repercusión en su presente y su futuro (Abellán López, 2021). El cambio climático antropogénico se produce por la emisión de gases efecto invernadero siendo el más abundante el $\mathrm{CO}_{2}$ que supone un $80,7 \%$ de las emisiones totales, seguido del metano con un $11,9 \%$, según el Inventario Nacional de Emisiones de Gases Efecto Invernadero 1990-2018, (Muntean et al., 2018). Consciente del alcance del problema, la Comisión Europea ha adoptado la primera ley climática de la Unión Europea para lograr la neutralidad climática en 2050. Asimismo, España adoptó el I Plan Nacional de Adaptación al Cambio Climático 2006-2020 y en estos momentos ya se ha implementado el II PNACC 2021-2030, que aspira a integrar todas las actuaciones de adaptación en los distintos terrenos de gestión pública y privada mediante 18 ámbitos de trabajo con diferentes objetivos para cada uno de ellos. Entre las grandes líneas de intervención se encuentra el diseño de programas educativos sobre alfabetización climática. La Convención Marco de las Naciones Unidas sobre el Cambio Climático (CMNUCC), a través de los Estados, está impulsando iniciativas de construcción de capacidades y elaboración de programas formativos sobre el cambio climático para lograr una mayor concienciación ciudadana. La crisis ecológica requiere acciones potentes para aumentar la alfabetización climática y la comprensión de la influencia humana en el calentamiento global (Cordero et al., 2008; Singh \& Mathur, 2019).

La generación de soluciones para cambiar las prácticas y las percepciones de la gente está influida por el contexto social y político que condiciona el éxito o fracaso de las intervenciones públicas. Conocer el impacto de cómo se comprenden los problemas resulta esencial para saber cómo las personas adaptan y modifican su conducta. En esta investigación se propone el estudio del nivel de cultura climática y de comportamientos individuales relativos al cambio climático en una muestra formada por alumnado y profesorado de ciencias sociales de la Universidad de Valencia y la Universidad de Alicante. 
Este trabajo busca una conexión compleja entre cambio climático y las pandemias, lo que ha sido puesto de manifiesto desde diferentes disciplinas. En esta línea, diferentes expertos subrayan una relación entre salud y diversidad ecológica y un papel de la contaminación como factor agravante. Asimismo, la relación entre la pandemia y los relatos alternativos resulta relevante, ya que perjudican la salud comunitaria con la divulgación de contenidos erróneos y dañinos como la denominada infodemia (Mancinas-Chávez, 2021) y la infoxitación (Innerarity, 2021). Este fenómeno también afecta a los centros de educación superior, tal como señalan autores como Solís \& Morón (2020).

\section{Marco Teórico}

\section{Los bienes comunes y el enfoque transversal de las Ciencias Sociales}

La necesidad de contar con programas transversales que incluyan contenidos medioambientales está estrechamente relacionada con los problemas de acción colectiva y las dificultades que presentan para abordarlos de forma individual.

La contaminación, la destrucción del medio natural y la formación de desequilibrios en la cadena son solo algunos ejemplos de los comportamientos "racionales" de los individuos. Siguiendo un razonamiento propio de la teoría de la elección racional, los productores de estas externalidades negativas se enfocan de manera exclusiva en su propio interés y con estas acciones generan ciertos fallos de mercado que de forma acumulativa están destruyendo el medio ambiente y provocando la situación conocida como la tragedia de los comunes (Hardin, 1968; Ostrom, 2015; Rifkin, 2019). El tratamiento de los temas sobre el medio natural desde la perspectiva del autointerés conduce a un agravamiento de los problemas y su destrucción, lo que perjudica a toda la humanidad. Esta confrontación entre el autointerés y el interés colectivo mantiene vínculos con lo que se denomina racionalidad limitada, en el sentido de que el ser humano no puede ni saber de todo, ni conocer todas las variables ni ser absolutamente racional (Simon, 2000). Además, los problemas medioambientales han sido caracterizados como wicked problems (Rittel \& Webber, 1973), es decir, problemas retorcidos o perversos que incluyen cuestiones complejas y visiones muy fragmentarias. Un problema retorcido se resiste a una definición unívoca y clara; además, cada intento por buscar soluciones cambia la formulación del mismo problema. Pensemos en problemas sistémicos como los medioambientales o los pandémicos que cuentan con interconexiones e interfaces extremadamente complejas y con dinámicas internas que superan las capacidades de comprensión y la previsión de riesgos. La búsqueda de soluciones tiene efectos colaterales que pueden generar problemas tan graves como el original dado que las soluciones que se obtienen se ajustan a una definición minimalista del problema. Por ello, los problemas climáticos deben considerarse asuntos públicos, lo que equivale a reconocer su complejidad, su fragmentación y su nivel de incertidumbre.

\section{La alfabetización como respuesta al desafío socio ambiental}

En este contexto, la alfabetización climática ha suscitado un enorme interés en las instituciones internacionales, en los gobiernos y en las administraciones educativas. Es un término que hace referencia a los marcos cognitivos y los valores que precisan las acciones de los individuos en su vida cotidiana en pro del medio ambiente. Así, la 
educación se convierte en un instrumento que sirve para dotar al alumnado de información y valores sobre la emergencia climática.

A través de diferentes programas, la cultura climática se ha erigido en la protagonista para ofrecer argumentos de calidad sobre las causas, los efectos y los riesgos socioambientales del calentamiento global y, a la vez, proporcionar información veraz para toda la ciudadanía. La educación, en cualquiera de sus niveles, resulta clave para dotar de competencias y conocimientos climáticos e influir en los comportamientos individuales. La formación en cultura climática capacita a las personas, en tanto consumidores y ciudadanos responsables y modela comportamientos y estilos de vida para abordar los problemas de sostenibilidad en nuestra era global interconectada. Las instituciones educativas son clave para la interiorización de nuevos valores y buenas prácticas en función de lo que es deseable socialmente (Scott, 1995).

Pero la lucha contra el cambio climático y la transición a una nueva economía menos contaminante requiere de todo el sistema de conocimiento de nuestra sociedad y las ciencias sociales juegan un papel esencial en ese sistema. Esta afirmación nos da una idea del alcance que pueden conseguir los esfuerzos hacia la alfabetización climática cuando se generalizan a las ciencias sociales, no solo a las ciencias físico-naturales, con su previsible transferencia a la sociedad.

\section{La necesidad de contar con enfoques integrados en la educación superior}

Los desafíos de la humanidad necesitan un enfoque integrado que debe incluir las ciencias sociales para enfrentar los problemas y buscar soluciones. Cualquier medida científica o política ha de tener en cuenta los factores sociales (normas culturales, educación, demografía, conflictos, redes sociales, parentesco, dinámicas de poder) para tener éxito en su implementación. Asegurar la eficacia de las políticas públicas requiere conocer el funcionamiento social de las cosas.

Las estrategias de lucha, mitigación y adaptación al cambio climático mantienen estrechos vínculos con los valores, con la política, con los comportamientos consumistas y con actitudes tanto individuales como colectivas. La preocupación por el medio ambiente, las consecuencias ecológicas y la sostenibilidad son un objetivo prioritario también para las ciencias sociales.

La educación es un componente imprescindible para adquirir capacidades adaptativas puesto que puede suministrar amplios conocimientos y habilidades para tomar decisiones informadas sobre cómo adaptar la vida y las circunstancias personales a los contextos cambiantes. La alfabetización climática concierne tanto a las enseñanzas no universitarias como las universitarias. Este enfoque ha de ser holístico, integrando Ciencias Sociales con una visión técnica, ya que esta última es insuficiente. No se trata de elegir sino de complementar y de brindar apoyo en un marco en que tenga cabida el conocimiento técnico y el conocimiento social.

Según la US Global Change Research (2009), la alfabetización climática es la comprensión de la influencia sobre el clima y cómo influye en cada uno de los individuos y en la sociedad. Todo ello implica: a) comprender los principios esenciales del sistema climático; b) capacitarse para evaluar información científica y veraz sobre el clima; c) comunicar con eficacia sobre el cambio climático y, d) tomar decisiones informadas y responsables sobre las acciones que afectan al clima. 


\section{La importancia de las fake news y la desinformación en un contexto de pandemia}

En este punto resulta oportuno referirse a la desinformación y a las fake news puesto que la cuestión climática ha sido el objetivo de campañas de desinformación durante décadas (Dunlap \& McCright, 2011; Van der Linden et al., 2017; Stevenson et al. 2017). Tanto la desinformación como las fake news responden a una estrategia para difundir de manera deliberada e intencional información no rigurosa que busca distorsionar la realidad, manipular la opinión pública y desestabilizar un estado de opinión. Como el cambio climático ha sido objeto de complejas tramas de desinformación, una de las respuestas que se articuló para enfrentarlo fue el movimiento de alfabetización climática que pretende una mejor comprensión del sistema climático (McGinnis et al., 2016; Shwom et al., 2017; Damico et al., 2018). Con la adquisición de ciertas competencias básicas y las herramientas conceptuales adecuadas se pretende que la gente esté en condiciones de evaluar la información sobre el cambio climático y tomar decisiones informadas y responsables.

Para lograr estos objetivos, Dupigny-Giroux (2010) afirma que es necesario que la alfabetización climática sea una actividad formativa a lo largo de toda la vida e identifica 6 retos importantes: 1) el manejo y la comprensión de la terminología específica de la ciencia climática; 2) el papel de las concepciones erróneas; 3) la incorporación en el currículo de todas las personas y no solo de estudiantes del ámbito científico; 4) la importancia de los estilos de aprendizaje; 5) la formación y las capacidades del profesorado y, 6) el papel de la experiencia vital de cada persona y las forma de acceso a la información.

Dado que el entorno educativo juega un papel determinante en la formación ciudadana, los acuerdos internacionales subrayan la necesidad de cooperar en la adopción de medidas destinadas a mejorar la educación, la sensibilización, la participación del público y el acceso público a la información. Prueba de ello es la Convención Marco de Naciones Unidas sobre Cambio Climático (art. 6), las Conferencias de las Partes de la Convención Marco de las Naciones Unidas sobre el Cambio Climático (COP20, Lima; COP21, Paris; COP25, Madrid), el Convenio de Aarhus, el Acuerdo de Escazú (para América Latina y Caribe), los Objetivos de Desarrollo Sostenible (ODS 4 y 13) de la Agenda 2030 y la Declaración de AichiNagoya sobre la Educación para el Desarrollo Sostenible de la UNESCO.

En función de este marco teórico se han generado las siguientes hipótesis de investigación:

- Hipótesis 1. La mayoría de encuestados tienen una sensibilidad manifiesta hacia los efectos del cambio climático y sus consecuencias en el ámbito social que se sustenta en su conocimiento sobre el tema y sus actitudes.

- Hipótesis 2. El conocimiento de las acciones públicas sobre el cambio climático es limitado, así como los efectos prácticos sobre tipos concretos de contaminación o fenómenos específicos.

- Hipótesis 3. La falta de confianza en la ciencia y sus recomendaciones es minoritaria en el ámbito de la educación superior. 


\section{Metodología}

Para su realización, este trabajo ha privilegiado una perspectiva descriptiva e interpretativa sobre los fenómenos político-sociales en detrimento de modelos estadísticos, tanto por la tipología de muestra y sus limitaciones como por el objetivo del trabajo. En concreto, se ha buscado testear un modelo de cuestionario y unas hipótesis de trabajo para comprobar si sus resultados pueden ser significativos para aplicar en futuras investigaciones. El instrumento utilizado fue un cuestionario ad-hoc online en Google Docs a estudiantes y profesorado de disciplinas de ciencias sociales de la Universidad de Valencia y de la Universidad de Alicante.

Antes de su lanzamiento, se debatió y verificó con un grupo formado por 5 profesores de sociología y ciencia política de la Universidad de Valencia, Universidad de Alicante y Universidad Miguel Hernández de Elche, cuyas aportaciones se incluyeron en el instrumento. Este cometido se concretó en la exploración del nivel de conocimientos sobre la cultura climática, así como las actitudes hacia el cambio climático, su relación con la crisis sanitaria y la puesta en duda de la labor de las instituciones y la investigación. Al igual que Morón \& Wamba (2010), se considera que las actitudes acercan a los investigadores a la práctica cotidiana de los individuos y de sus concepciones, por lo que su estudio también ayuda a generar conocimiento sobre sus percepciones. El estudio se llevó a cabo a través de una metodología cuantitativa que se concretó en ítems con respuesta cerrada y semicerrada y el uso de métodos de estadística descriptiva. Esta permite observar el fenómeno social estudiado en función de un marco teórico previo a través del método hipotético-deductivo. La encuesta, en consecuencia, sirve al propósito de estudiar la realidad estudiada y comprobar empíricamente las hipótesis de investigación propuestas (Corbetta, 2010). En concreto, todos estos objetivos se concretaron en las siguientes preguntas, tal como se incluyen en la Tabla 1.

Tabla 1. Cuestionario. Fuente: Elaboración propia.

\begin{tabular}{ll}
\hline Variable & Pregunta \\
\hline Edad & Grupo de edad al que perteneces \\
\hline Sexo & Sexo \\
\hline Procedencia & Código Postal \\
\hline Estudios & ¿Cuál es tu nivel de estudios? \\
\hline Cambio climático & ¿Sabes en qué consiste el cambio climático? \\
\hline $\begin{array}{l}\text { Calentamiento global - } \\
\text { naturales }\end{array}$ & $\begin{array}{l}\text { El calentamiento global está ocurriendo solo debido a } \\
\text { factores naturales y no se puede evitar. }\end{array}$ \\
\hline $\begin{array}{l}\text { Calentamiento global - } \\
\text { humanas }\end{array}$ & $\begin{array}{l}\text { Las emisiones de gases efecto invernadero de nuestras } \\
\text { actividades diarias son responsables del cambio climático }\end{array}$ \\
\hline $\begin{array}{l}\text { Cop25 } \\
\text { En diciembre de 2019 se celebró en Madrid la CoP25, ¿sabes } \\
\text { de qué se trató? }\end{array}$ \\
\hline
\end{tabular}


Tabla 1. Continuación.

\begin{tabular}{|c|c|}
\hline Variable & Pregunta \\
\hline Participación climática & $\begin{array}{l}\text { ¿En los últimos } 12 \text { meses has asistido a alguna charla o curso } \\
\text { sobre el cambio climático? }\end{array}$ \\
\hline Friday for Climate Change & $\begin{array}{l}\text { ¿Sabes en qué ha consistido la iniciativa Friday for Climate } \\
\text { Change? }\end{array}$ \\
\hline Efecto Invernadero & ¿Entiendes en qué consiste el efecto invernadero? \\
\hline Gases & $\begin{array}{l}\text { Si ha contestado sí a la anterior, ¿Puedes indicar alguno de } \\
\text { los gases que produce el efecto invernadero? }\end{array}$ \\
\hline Bosques & $\begin{array}{l}\text { La protección de los bosques y la forestación contribuyen a la } \\
\text { mitigación del cambio climático. }\end{array}$ \\
\hline Plásticos & $\begin{array}{l}\text { Los plásticos biodegradables contribuyen al calentamiento } \\
\text { global y al cambio climático }\end{array}$ \\
\hline Energía Renovable & $\begin{array}{l}\text { El uso de fuentes de energía renovables puede contribuir a la } \\
\text { mitigación del cambio climático }\end{array}$ \\
\hline Riesgo Vida Humana & $\begin{array}{l}\text { El cambio climático plantea algún riesgo para la vida } \\
\text { humana, animal y vegetal }\end{array}$ \\
\hline España Riesgo & España está en riesgo por el cambio climático. \\
\hline Acuerdo de París & ¿Sabes si España firmó el Acuerdo de París? \\
\hline $\begin{array}{l}\text { Panel Intergu- } \\
\text { bernamental }\end{array}$ & $\begin{array}{l}\text { ¿Conoces el Panel Intergubernamental sobre Cambio } \\
\text { Climático? }\end{array}$ \\
\hline Plan Nacional & $\begin{array}{l}\text { ¿Sabes qué es el Plan Nacional de Adaptación al Cambio } \\
\text { Climático? }\end{array}$ \\
\hline Políticas Públicas & $\begin{array}{l}\text { ¿Conoces alguna iniciativa pública para mitigar el cambio } \\
\text { climático? }\end{array}$ \\
\hline Administración & $\begin{array}{l}\text { En caso de responder afirmativamente a la pregunta } \\
\text { anterior, ¿puedes indicar de qué administración pública se } \\
\text { trata? }\end{array}$ \\
\hline Comportamiento & $\begin{array}{l}\text { Tu comportamiento de consumo individual afecta a las } \\
\text { emisiones totales de gases de efecto invernadero de España }\end{array}$ \\
\hline Medida & $\begin{array}{l}\text { ¿Tomas alguna medida a nivel individual para reducir las } \\
\text { emisiones de gases de efecto invernadero? }\end{array}$ \\
\hline Tipos de medida & $\begin{array}{l}\text { En caso de responder afirmativamente a la anterior, ¿puedes } \\
\text { señalar alguna? }\end{array}$ \\
\hline Esfuerzo & $\begin{array}{l}\text { Harías un esfuerzo para tomar decisiones con conciencia } \\
\text { ambiental si conocieses mejor el impacto de tus acciones en } \\
\text { el medio ambiente }\end{array}$ \\
\hline
\end{tabular}


Tabla 1. Continuación.

\begin{tabular}{|c|c|}
\hline Variable & Pregunta \\
\hline Alarmismo & $\begin{array}{l}\text { Piensas que se exagera un poco sobre esta cuestión y hay } \\
\text { mucho alarmismo }\end{array}$ \\
\hline COVID19 & $\begin{array}{l}\text { La crisis sanitaria del Coronavirus es tan importante como la } \\
\text { lucha contra el cambio climático }\end{array}$ \\
\hline COVID correlación & $\begin{array}{l}\text { La situación de pandemia ayudará a concienciar a las } \\
\text { personas sobre los riesgos climáticos }\end{array}$ \\
\hline Gobiernos & $\begin{array}{l}\text { Los gobiernos deberían actuar con la misma contundencia } \\
\text { ante el cambio climático como con el Covid-19. }\end{array}$ \\
\hline Pandemias y cambio climático & $\begin{array}{l}\text { El cambio climático y la pérdida de biodiversidad aceleran la } \\
\text { propagación de enfermedades infecciosas. }\end{array}$ \\
\hline Cambio social Covid-19 & $\begin{array}{l}\text { Van a cambiar muchos hábitos sociales cuando la sociedad } \\
\text { supere la crisis sanitaria del Covid-19. }\end{array}$ \\
\hline Confinamiento & $\begin{array}{l}\text { El confinamiento en casa y la reducción de nuestra } \\
\text { movilidad ha tenido consecuencias ambientales positivas. }\end{array}$ \\
\hline Corresponsabilidad & $\begin{array}{l}\text { La crisis sanitaria del Covid-19 ha fortalecido la conciencia } \\
\text { de colectividad y la corresponsabilidad social. }\end{array}$ \\
\hline Ciencia & $\begin{array}{l}\text { La ciencia y la confianza en las instituciones son } \\
\text { fundamentales para combatir los riesgos globales como la } \\
\text { pandemia y el cambio climático }\end{array}$ \\
\hline Medios de comunicación & $\begin{array}{l}\text { Para estar informado sobre los problemas climáticos y la } \\
\text { pandemia el medio de información utilizado } \\
\text { preferentemente }\end{array}$ \\
\hline
\end{tabular}

Este cuestionario de 36 preguntas fue enviado a un total de 400 personas, entre alumnado de grado y posgrado, becarios y profesorado de ciencias sociales y estuvo disponible del 1 de abril al 7 de mayo de 2020. Estos ítems recopilaron información en cuatro bloques diferenciados. El primer bloque se componía de 4 preguntas sobre información sociodemográfica. El segundo bloque contaba con 17 preguntas sobre conocimientos generales de cultura climática y política ambiental. El tercer bloque, con 5 preguntas, indagaba sobre actitudes y comportamiento hacia el consumo individual. El cuarto y último bloque planteaba 9 preguntas sobre la relación percibida entre cambio climático y Covid-19. De la invitación a participar se consiguieron 237 respuestas lo que supone una tasa de respuesta del 59,25\%.

El carácter exploratorio de este trabajo pretende desarrollar una inferencia parcial y limitada para comprobar que el enfoque utilizado puede ser aplicado a una población más amplia en futuras investigaciones. Si bien las respuestas obtenidas, así como la conformación de la muestra no permiten una generalización de los resultados a todo el colectivo, entendemos que sí ofrecen cierto valor interpretativo 
que merece ser considerado. La percepción de las personas encuestadas genera valor científico tanto por las circunstancias en las que se ha producido el estudio como por la temática específica de la pandemia y su relación con el medio ambiente.

\section{Resultados}

\section{Variables sociodemográficas de los encuestados}

Las principales variables sociodemográficas solicitadas en la investigación eran el grupo de edad, el sexo, el código postal y el nivel de estudios. Un 48,5\% de encuestados pertenecía al grupo de edad más joven, comprendido en la franja de entre 18 a 30 años, lo que se explica porque corresponde a estudiantes universitarios. Por sexos, la mayoría de participantes han sido mujeres, con un $60,8 \%$, mientras que los hombres han representado un 39,2\%. Por niveles de estudios, un $38,4 \%$ respondió que su nivel de estudios alcanzado era bachillerato seguido de un $30 \%$ que era licenciado o graduado, lo que se explica por los alumnos que cursaban el máster o doctorado. También participaron en la encuesta un 4,2\% de personas, cuyo máximo nivel académico era graduado en ESO, lo que concuerda con el alumnado matriculado en los programas de acceso de mayores de 25 años y de 45 años. Respecto a los doctores, el porcentaje de participación fue del 15,2\%. En cuanto a la procedencia geográfica, encontramos participantes de la Comunidad Valenciana de manera preeminente pero también de otras comunidades autónomas como Murcia, Andalucía, Madrid, Castilla-La Mancha, Cataluña, Baleares, Canarias e incluso de ámbito internacional, debido fundamentalmente a que buscaron confinarse en sus hogares aunque cursaran sus estudios en la Universidad de Valencia o en la Universidad de Alicante.

Tabla 2. Participantes por grupos de edad y sexo.

\begin{tabular}{llllllll}
\hline Categorías & $\mathbf{1 8 - 3 0}$ & $\mathbf{3 1 - 4 0}$ & $\mathbf{4 1 - 5 0}$ & $\mathbf{5 1 - 6 5}$ & $\mathbf{6 6 - 7 5}$ & $\boldsymbol{>} \mathbf{7 6}$ & Total \\
\hline Hombre & 38 & 10 & 12 & 18 & 13 & 2 & 93 \\
\hline Mujer & 77 & 12 & 15 & 27 & 12 & 1 & 144 \\
\hline Total general & $\mathbf{1 1 5}$ & $\mathbf{2 2}$ & $\mathbf{2 7}$ & $\mathbf{4 5}$ & $\mathbf{2 5}$ & $\mathbf{3}$ & $\mathbf{2 3 7}$ \\
\hline
\end{tabular}

\section{Análisis de los conocimientos sobre cultura climática y políticas ambientales en España}

Respecto a la cultura climática, el $100 \%$ de los encuestados posee conocimientos sobre el calentamiento global y el cambio climático. Se preguntó si las emisiones de gases efecto invernadero de nuestras actividades son responsables del cambio climático y un 49,9\% estuvo muy de acuerdo y un 32,5\% se mostró de acuerdo con esta afirmación. Solo un 11,8\% se mantuvo en la posición intermedia de la escala, un $2,5 \%$ se mostró en desacuerdo y un 3,8\% muy en desacuerdo. Un 90,3\% de los encuestados manifestaron una clara comprensión del fenómeno y supieron identificar los principales gases contaminantes en la pregunta abierta. La mayoría de los sujetos encuestados destacaron como principal gas efecto invernadero el dióxido de carbono o $\mathrm{CO} 2$, seguido por el gas metano, el ozono y el dióxido de nitrógeno. Un 
96,6\% destacó que el cambio climático es un fenómeno que pone en peligro la vida del planeta y un $91,1 \%$ entiende que también pone en riesgo la supervivencia de España. Asimismo, un $88,1 \%$ de los encuestados piensa que los bosques son un elemento fundamental para luchar contra el calentamiento global y un 89,1\% estima que las renovables son energías que pueden ayudar a combatirlo. En relación con los plásticos biodegradables, solo un 32,9\% los consideró un problema. Este bajo porcentaje y que la mayoría de las respuestas se situaran en el ítem intermedio de la escala, nos lleva a considerar la posibilidad de cierto desconocimiento sobre los efectos de los plásticos y su proceso de fabricación para el medio ambiente.

Respecto al conocimiento sobre las acciones políticas no convencionales y de participación del alumnado y profesorado de ciencias sociales en actos reivindicativos, solo un $30 \%$ de los encuestados señala que ha participado activamente de este tipo de acciones. Respecto a acciones concretas de defensa del medio ambiente, un $42,9 \%$ de los encuestados desconocía la iniciativa Friday for Climate Change y solo un 16,5\% de los que la conocían había participado de ella.

Sobre el nivel de conocimientos de las acciones adoptadas por las autoridades españolas, un 34,6\% no sabe si España ratificó o no el acuerdo de París. Resulta significativo que el $81,4 \%$ de los encuestados no conozca qué es el Panel Intergubernamental sobre el Cambio Climático; que un 38,8\% desconozca de qué trató la COP 25 celebrada en Madrid en diciembre de 2019 y que un 64,1\% no sepa qué es el Plan Nacional de Adaptación al Cambio Climático. Sin embargo, un 62,9\% manifiesta conocer alguna iniciativa para luchar contra el cambio climático y el nivel político administrativo en el que se ha implantado. Un 34,7\% de las personas encuestadas considera que el Estado es quien realiza el mayor esfuerzo climático seguido de un 30,6\% que opta por la administración local.

\section{Análisis de las actitudes hacia el comportamiento del consumo individual}

En cuanto a las actitudes de comportamiento individual y de preservación del medio ambiente, se puede destacar que más del $60,7 \%$ de los encuestados intenta minimizar sus impactos en las emisiones de gases. Existe una clara percepción de los peligros del calentamiento global y sus efectos perniciosos para la vida humana, representada con un $81,1 \%$ de respuestas. De hecho, un $78,1 \%$ ha puesto en marcha medidas individuales domésticas para mitigar el cambio climático. Sin embargo, hay una pequeña parte de respuestas de personas encuestadas que no están dispuestas a cambiar de hábitos de vida.

La mayoría de las medidas se vinculan con tres ejes fundamentales: 1) el transporte, 2) las nuevas formas de consumo y 3) la relación del ser humano con los residuos. Entre las medidas relacionadas con el transporte, se incidió preferentemente en el uso del transporte público y del transporte sostenible (bicicleta, marcha a pie). Asimismo, también se mencionaron la utilización de vehículos con una menor huella de carbono como los eléctricos, los híbridos, las motos eléctricas de alquiler o el carsharing.

Respecto a las medidas sobre nuevas formas de consumo aparecen dos tendencias destacables. Por un lado, la disminución de consumo de productos como ropa o el ahorro en suministros como la energía eléctrica y la no adquisición de productos de plástico o aerosoles. Por el otro, encontramos patrones de consumo que abogan por 
la producción ecológica y de proximidad ética y sostenible. A estas tendencias las acompaña el cambio de hábitos alimentarios a través de la reducción de la ingesta de carne en distinto grado, la producción y uso de energías renovables, así como el cultivo personal de vegetales o el autoconsumo. Finalmente, las medidas relacionadas con la gestión y tratamiento de los residuos son de las más mencionadas por los encuestados. De hecho, el reciclaje, la reutilización y la minimización son acciones clave para las personas encuestadas. Incluso algunas respuestas sostienen la formación de compost con sus propios residuos para el cultivo y cuidado de plantas. Lo cierto es que un $86,9 \%$ considera que un mejor conocimiento del tema facilitaría una optimización de sus decisiones climáticas.

\section{Análisis de la relación entre cambio climático y Covid-19}

Algunas instituciones expertas como la Organización Mundial de la Salud (OMS), la OCDE y el Informe Global Risks Report (2020) sostienen que la proliferación de la Covid-19 guarda una relación directa con el cambio climático. Siguiendo esta idea, la presente investigación incluyó en el cuestionario un bloque de preguntas que relacionaban cambio climático y Covid-19. El contexto de confinamiento obligado por la pandemia ofrecía la oportunidad y la pertinencia para explorar las percepciones sobre esta inédita situación.

A la pregunta de si la crisis sanitaria era percibida tan importante como la lucha contra el cambio climático, un 48,5\% de los encuestados valoraron con un 5 esta afirmación (puntuación más alta de la escala) y un 28,3\% con un 4 . Es decir, una mayoría de los encuestados otorgaba la misma importancia a la lucha contra la pandemia y al cambio climático. Solo un $16,5 \%$ se ubicó en una posición intermedia y apenas un 6,8\% se mostró poco o nada de acuerdo.

La segunda pregunta de este bloque indagaba si la situación de pandemia ayudaría a concienciar a las personas sobre los riesgos climáticos. La valoración a esta pregunta muestra una clara división de respuestas diferenciadas. Un 38,4\% de encuestados se mostró muy de acuerdo o de acuerdo con que la situación de pandemia ayudaría a concienciar sobre el riesgo climático.

Un 30,8\% se ubicó en la posición intermedia de la escala y un 30,5\% de las personas encuestadas se mostró poco o nada de acuerdo con que la pandemia contribuiría a concienciar a las personas sobre los riesgos climáticos.

Interrogados sobre si los gobiernos deberían actuar con la misma contundencia ante el cambio climático como con la Covid-19, un 87,8\% se mostró de acuerdo o muy de acuerdo con esta afirmación, de lo que se infiere que existe una percepción de que los poderes públicos deberían aplicar enfoques de más alcance y políticas públicas más ambiciosas en la lucha contra el cambio climático. Solo un 8,4\% respondió que no estaba ni de acuerdo ni en desacuerdo y un 3,8\% se mostró en desacuerdo o muy en desacuerdo con esta afirmación.

También se preguntó si el cambio climático aceleraba la propagación de enfermedades infecciosas, de los que un 68,8\% se mostró de acuerdo o muy de acuerdo con esta afirmación mientras que un $24,1 \%$ respondió que no estaba ni de acuerdo ni en desacuerdo. Solo un 7,2\% se mostró en desacuerdo entre la relación entre cambio climático y propagación de enfermedades infecciosas. 
Otra de las preguntas del cuestionario abordaba el cambio de hábitos para la nueva normalidad. Las respuestas mayoritarias se concentraron en la posición media de la escala, con un 39,2\% que respondió que no estaba ni de acuerdo ni en desacuerdo. La ausencia de un alineamiento explícito puede explicarse bien por desconocimiento o por falta de identificación o de resistencia ante los cambios. Un $20,7 \%$ se mostró muy de acuerdo y un 23,6\% simplemente de acuerdo con esta afirmación. El porcentaje de encuestados que perciben que los hábitos sociales van a cambiar poco o muy poco suponen el $11,4 \%$ y el $5,1 \%$ respectivamente.

El cuestionario incluía una pregunta sobre el grado de confianza en la ciencia y en las instituciones para combatir los riesgos globales, como la pandemia y el cambio climático. Las respuestas recibidas confirman que un $86 \%$ de los encuestados estaba muy de acuerdo o de acuerdo con la necesidad de confiar en las instituciones mientras que un $9,7 \%$ no estaba ni de acuerdo ni en desacuerdo y solo un $4,2 \%$ respondió que estaba en desacuerdo. Cuando se preguntó si el confinamiento y la reducción de la movilidad habían tenido consecuencias ambientales positivas, un $96,2 \%$ respondió estar muy de acuerdo o de acuerdo con esa afirmación. El resto de las respuestas de la escala a nivel agregado no alcanzó ni un 4\% de respuestas.

Respecto a si la crisis sanitaria había fortalecido la conciencia de colectividad y la corresponsabilidad social, un 63,8\% estuvo muy de acuerdo o de acuerdo con esta afirmación. Un 26,6\% respondió que no estaba ni de acuerdo ni en desacuerdo. Esto puede explicarse por la duda generalizada de que existan miembros de la colectividad que antepongan el interés común al suyo propio, un problema que ya se planteó en las referencias teóricas de este trabajo y que forma parte de la lógica de la acción colectiva y de la tragedia de los comunes. Solo un 9,7\% de los encuestados respondió que de la experiencia colectiva de la pandemia no se deriva necesariamente un fortalecimiento de la conciencia colectiva y de la corresponsabilidad social.

La última pregunta del cuestionario interrogaba sobre las fuentes de información que han sido consultadas para informarse sobre la pandemia. En la pregunta se obligaba a los encuestados a descantarse por un solo medio. La mayoría de los encuestados, con un 38,4\%, destacó la prensa como fuente principal, seguida de las redes sociales y las notificaciones personalizadas con un 29,5\%. A poca distancia, pero relegada a un tercer lugar, aparece la televisión con un 24,9\%. La última del ranking es la radio con un $7,2 \%$.

\section{Discusión}

Los resultados de este artículo ponen de manifiesto que, en el ámbito universitario objeto de estudio, existe plena consciencia de la problemática del cambio climático, de su naturaleza y sus causas. Sin embargo, respecto a la participación, existe una doble visión según afecte a la esfera individual o al elemento de acción colectiva.

Así, la mayoría de los encuestados destacan que toman decisiones para paliar la huella de carbono que generan a través de métodos ecológicos o colectivos de transporte, la autogeneración de energía o el reciclaje, entre otros. No obstante, a la hora de reivindicar el cambio de modelo productivo, son pocos los que conocen las iniciativas de reivindicación, así como los instrumentos puestos en marcha por las instituciones públicas. Esta forma de proceder sugiere la falta de identificación de 
parte de los encuestados con los instrumentos públicos y de acción colectiva para la defensa del medio ambiente. A tenor de los resultados, parece que las acciones climáticas tienen éxito cuando se abordan desde el comportamiento individual, cuando de lo que se trata es de llegar a una visión colectiva que es más costosa de conseguir.

Respecto a la relación entre cambio climático y Covid 19, la mayoría de las personas encuestadas señala la existencia de una estrecha relación entre ambos fenómenos y entiende la necesidad de articular medidas públicas para mitigar los efectos de la pandemia y del cambio climático. En este bloque de preguntas se detectó un aumento de respuestas en el nivel intermedio de la escala, lo que se interpreta como un posicionamiento de indefinición para soslayar preguntas sobre las que no se tiene un alineamiento claro. Esta posición intermedia es mayoritaria cuando se pregunta por el nivel de conciencia sobre los efectos climáticos y la pandemia o sobre el cambio de hábitos sociales después de la crisis sanitaria.

Aunque la gran mayoría tiene una opinión positiva sobre el papel de la ciencia, destaca un porcentaje del 3,4\% que piensa que el cambio climático es producido por causas naturales. Este porcentaje sube al 6,33\% para aquellos que están en desacuerdo con que el ser humano sea el causante. Con estos datos, se puede afirmar un cierto discurso alternativo que cuestiona la importancia del problema y una falta de conciencia de sus efectos a corto plazo.

\section{Conclusiones}

Este trabajo ha presentado los resultados de una encuesta que se ha distribuido entre alumnado y profesorado de dos universidades públicas españolas, la Universidad de Valencia y la Universidad de Alicante. Con esta investigación se pretendió generar evidencias sobre las actitudes y conocimientos de la cultura climática sobre una muestra de conveniencia centrada en alumnado y profesorado de educación superior.

Como se ha podido comprobar, la mayoría de los encuestados reconocen la existencia del cambio climático y de los desafíos globales como la Covid-19. Los resultados señalan la existencia de cierto grado de concienciación para adoptar comportamientos responsables con compromiso variable entre los encuestados, lo que indica que se cumple la hipótesis 1.

Asimismo, la mayoría de encuestados manifiesta conocer alguna medida puestas en marcha por los poderes públicos, pero de las respuestas no se desprende que exista un conocimiento experto y concreto. Esta afirmación queda de manifiesto por las dificultades para reconocer, por ejemplo, la COP25 o los Friday for Planet. Respecto a las relaciones entre Covid-19 y cambio climático, la mayoría de las personas encuestadas percibe que existe una relación directa y que el compromiso y la contundencia en la lucha contra la crisis sanitaria deberían hacerse extensivos a la cuestión climática. De esta forma, se puede afirmar que se cumple parcialmente la hipótesis 2.

Una de las evidencias más interesantes es que la mayoría de la muestra piensa que la ciencia es fundamental y que su papel para solucionar problemas es importante. 
Solo una pequeña minoría considera que las contribuciones científicas no son efectivas y por ello se puede afirmar que se cumple la hipótesis 3.

Como se ha mencionado anteriormente, si bien la muestra de este trabajo es limitada y no permite la generalización, ello no le resta valor.

Entre otras líneas futuras de investigación se apunta un mayor conocimiento de cómo los estudiantes y el profesorado perciben la sostenibilidad curricular y su impacto en los planes de estudio de las ciencias sociales y humanas.

\section{Referencias bibliográficas}

Abellán López, M. A. (2021). Capital social, redes de confianza y cambio climático. Un enfoque neoinstitucionalista-tecnocrático. Miríada, 13(17), 251-269.

Cordero, E., Todd, A. M. \& Abellera, D. (2008). Climate change education and the ecological footprint. Bulletin of American Meteorological Society, 89(6), 865872. https://doi.org/10.1175/2007BAMS2432.1

Corbetta, P. (2010). Metodología y Técnicas de Investigación Social. (Edición Revisada). McGraw-Hill.

Damico, J., Baildon, M., \& Panos, A. (2018). Media Literacy and Climate Change in a Post-Truth Society. Journal of Media Literacy Education, 10(2), 11-32. https://doi.org/10.23860/JMLE-2018-10-2-2

Dunlap, R., \& McCright, A. (2011). Organized Climate Change Denial. En J. Dryzed, R. Norgaard, \& D. Schlosberg (eds.), Oxford Handbook of Climate Change and Society. Oxford University Press.

Dupigny-Giroux, L.-A. L. (2010). Exploring the challenges of climate science literacy: Lessons from students, teachers and lifelong learners. Geography Compass, 4(9), 1203-1217. https://doi.org/10.1111/j.1749-8198.2010.00368.x

Hardin, G. (1968). The tragedy of the commons. Science, 162(3859), 1243-1248. https://doi.org/10.1126/science.162.3859.1243

Innerarity, D. (2021). La pandemia de los datos. Dilemata, 13(35), 67-72. https://www.dilemata.net/revista/index.php/dilemata/article/view/412000 403/730

Mancinas-Chávez, R. (2021). De la pandemia a la infodemia: la proliferación de bulos en el tratamiento informativo del Coronavirus. En R. Mancinas-Chávez, \& M. L. Cárdenas-Rica (Eds.), Medios y comunicación en tiempos de posverdad. Fragua.

McGinnis, R., McDonald, C., Hestness, E., \& Breslyn, W. (2016). An Investigation of Science Educators' View of Roles and Responsibilities for Climate Change Education. Science Education International, 27(2), 179-192. https://files.eric.ed.gov/fulltext/EJ1104645.pdf

Morón-Monge, H. y Wamba Aguado, A. M. (2010). La Percepción sobre los Riesgos Ambientales como Indicador de los Obstáculos y Dificultades para la Construcción de un Concepto de Medio Ambiente Responsable. Bio-grafía, escritos sobre la biología y su enseñanza, 3(4), 114-137. https://revistas.pedagogica.edu.co/index.php/bio-grafia/article/view/340/822 
Muntean, M., Guizzardi, D., Schaaf, E., Crippa, M., Solazzo, E., Olivier, J. G. J., Vignati, E. (2018). Fossil CO2 emissions of all world countries - 2018 Report, EUR 29433 EN, Publications Office of the European Union, Luxembourg. https://doi.org/10.2760/30158

Ostrom, E. (2015). Governing the commons: The evolution of institutions for collective action. Cambridge University Press

Rifkin, J. (2019). Green New Deal Global. Paidós.

Rittel, H. W. J., \& Webber, M. M, (1973). Dilemmas in a general theory of planning. Policy Sciences, 4(2), 155-169. https://www.sympoetic.net/Managing_Complexity/complexity_files/1973\% 20Rittel\%20and\%20Webber\%20Wicked\%20Problems.pdf

Scott, W. R. (1995). Institutions and organizations. Sage Publications.

Singh, P., K. D., \& Mathur, A. (2019). Climate Literacy and Individual Consumption Behavior: An Evaluation of the Indian Experience. European Journal of Sustainable Development, 8(5), 187. https://doi.org/10.14207/ejsd.2019.v8n5p187

Schleicher, A., Zimmer, K., Evans, J., \& Clements, N. (2009). PISA 2009 Assessment Framework: Key Competencies in Reading, Mathematics and Science. OECD Publishing (NJ1).

Simon, H. A. (2000). Bounded rationality in social science: Today and tomorrow. Mind \& Society (1), 25-39. https://doi.org/10.1007/bf02512227

Shwom, R., Isenhour, C., Jordan, R., McCright, A., \& Meta-Robison, J. (2017). Integrating the social sciences to enhance climate literacy. Frontiers in Ecology and the Ennvironment, 15(7), 377-384. https://doi.org/10.1002/fee.1519

Solís-Espallargas, C., \& Morón-Monge, H. (2020). How to Improve Sustainability Competences of Teacher Training? Inquiring the Prior Knowledge on Climate Change in Primary School Students. Sustainability, 12(16), 6486. https://doi.org/10.3390/su12166486

Stevenson, R., Nicholls, J., \& Whitehouse, H. (2017). What is climate change education? Curriculum $\quad$ Perspectives, 37(1), 67-71. https://doi.org/10.1007/s41297-017-0015-9

The Global Risks Report (2020). World Economic Forum's https://es.weforum.org/reports/the-global-risks-report-2020

US Global Change Research (2009). Climate Literacy. The essential principles of Climate Science. https://downloads.globalchange.gov/Literacy/climate_literacy_highres_engli sh.pdf

Van der Linden, S., Leiserowitz, A. Rosenthal, S., \& Maibach, E. (2017). Inoculating the Public against Misinformation about Climate Change. Global Challenges, 1(2), 1-7. https://doi.org/10.1002/gch2.201600008 